\title{
How far down to push the LDL cholesterol? PCSK9 where statins fail
}

\author{
Simko $\mathrm{V}^{1}$, Ginter $\mathrm{E}^{2}$
}

State University New York, Downstate Medical Center at Brooklyn, USA. ginter.emil@mail.t-com.sk

Statins, an effective medication to lower cholesterol have been used for years, all around the world. Still, a proportion of patients do not respond to statins. Intensive research in the past decade (1) produced a new „wonder“ drug: PCSK9 inhibitor was approved by the US. Food and Drug Administration in July 2015. We have previously reported in this journal on novel management of high cholesterol, on PCSK9 and its role in cholesterol metabolism $(2,3,4,5)$.

PCSK9 will be marketed in the USA by Sanofi and Regeneron as Praluent (alirocumab). Repatha (evolocumab) developed by Amgen is expected to be approved soon $(6,7)$. Repatha and Praluent were approved in Europe, with less restrictive indications than in the USA. The estimates predict eight to ten million Americans in need to lower their cholesterol below the effect of a statin.

PCSK9 in the USA is indicated for patients who have had heart attacks (myocardial infarction), stroke, persistent coronary artery insufficiency or a genetic predisposition to high cholesterol, familial hypercholesterolemia (FH). Cardiologists at the prominent Johns Hopkins University claim „there is a high need for this medication".

$\mathrm{FH}$ is related to the mutation of a gene responsible for the receptor of low density cholesterol. Patients affected with FH who are 20 - 39 years of age have hundred times higher risk of ischemic heart disease, compared with non- FH individuals

Research in the past years revealed another important factor that is instrumental in interaction of LDL particled with the LDL receptor located at the surface of liver cells (hepatocytes). This receptor is a proprotein, an enzyme decisive in cholesterol regulation. It acts as a convertase subtilisin/kexin type 9 (PCSK9). The presence of PCSK9 at the surface of a hepatocyte inhibits the LDL inside the liver cell to dissociate and to return to the surface of the cell. This results in the rise of circulating LDL cholesterol.

Patients with FH have an inherited metabolic disorder that leads to extremely high cholesterol and increased complications of atherosclerosis. There are estimated 600 thousand Americans with FH who would be prime candidates to benefit from PCSK9, to bring their LDL to a ,safe“ level.

${ }^{1}$ State University New York, Downstate Medical Center at Brooklyn, USA, and ${ }^{2}$ Emeritus, Slovak Medical University, Bratislava, Slovakia

Address for correspondence: V. Simko MD, PhD, State University New York, Downstate Medical Center at Brooklyn, USA.

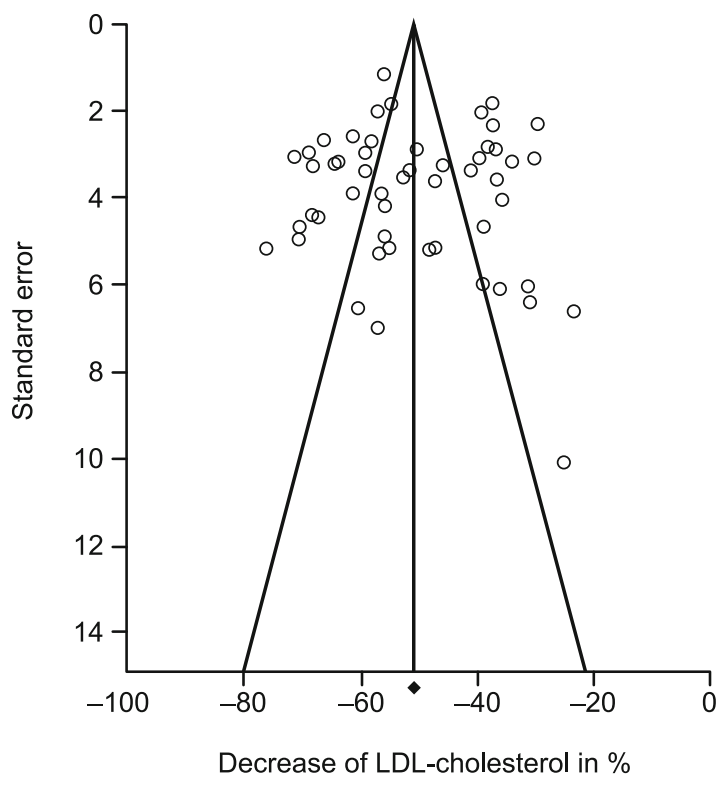

Fig. 1. Decrease of LDL-cholesterol after application of PC SK9 antibodies (adapted from Ann Intern Med 2015; 163 (1): 40-45).

There is considerable debate as how broadly this medication should be used. Some experts are worried about possible long-term safety. They insist that the medical community should not treat a laboratory value, aiming at the lowest possible blood level of LDL C. A meta analysis (6) comprising 10,169 individuals who were prescribed PCSK9, documented marked lowering of LDL C by almost 50 per cent (Fig. 1). Cardiovascular mortality decreased and there were no prominent side effects.

While clinical trials so far did not show signficant side effects (8), larger studies aimed at showing that PCSK9 prevents myocardial infarction and stroke are expected to bring decisive results only after 2017.

There is a concern that an expensive new treatment may disproportionately burden available health care funding. The insurance system is worried about wide, unrestricted prescribing of medication that may have to be taken for life. They estimate it may cost in the USA up to 10 billion dollars a year.

In contrast to orally administered statins, a monoclonal antibody like Praluent has to be self- injected every two weeks at 


\section{3-194}

\$ 560 per injection, with an estimaterd cost about \$ 14,600 per year.

The health plans intend to keep most patients on statins which were confirmed to prevent heart attacks. Statins are now mostly marketed as inexpensive generics. Some patients probably fail cholesterol lowering by a statin because they do not take it regularly. The health insurance system is considering to deliver prescribed statins by mail, to increase their effectiveness and to prevent switching to a new, much more costly medication.

\section{References}

1. Lagace TA. PCSK9 and LDLR degradation: regulatory mechanisms in circulation and in cells. Curr Opin Lipidol 2014; 25 (5): 387-393.

2. Ginter E, Simko V. New promising potential in fighting atherosclerosis: HDL and reverse cholesterol transport. Bratisl Lek Listy 2013; 114: 169-173.

3. Ginter E, Simko V. Update on medical management of dyslipidemia and atherosclerosis. Bratisl Lek Listy 2013; 114: 262-268.
4. Simko V, Ginter E. Understanding cholesterol: high is bad but too low may also be risky - is low cholesterol associated with cancer? Bratisl Lek Listy 2014; 115: 59 -65.

5. Ginter E, Simko V. New weapon in fighting atherosclerosis: Effective inhibition of circulating low density lipoprotein cholesterol. Bratisl Lek Listy 2014; 115: 741 - 742.

6. Navarese EP, Kolodziejczak M, Schulze V et al. Effects of Proprotein Convertase Subtilisin/Kexin Type 9 Antibodies in adults with hypercholesterolemia: A systematic review and meta-analysis. Ann Intern Med 2015; 163 (1): 40-51.

7. Sabatine MS, Giugliano RP, Wiviott SD et al. Open-label study of cholesterol (OSLER) Investigators. Efficacy and safety of evolocumab in reducing lipids and cardiovascular events. N Engl J Med 2015; 372: 1500-1509.

8. Keraiakes DJ, Robinson JG, Cannon CP et al. Efficacy and safety of the paraprotein convertase subtilisin/kexin type inhibitor alirocumab among high cardiovascular risk patients on maximally tolerated statin therapy. Heart J 2015; 169: 906-915.

Received August 20, 2015. Accepted February 